\title{
Pulsed Dual Electrospray Ionization for Ion/Ion Reactions
}

\author{
Yu Xia, Xiaorong Liang, and Scott A. McLuckey \\ Department of Chemistry, Purdue University, West Lafayette, Indiana, USA
}

\begin{abstract}
A pulsed dual electrospray ionization source has been developed to generate positive and negative ions for subsequent ion/ion reaction experiments. The two sprayers, typically a nano-electrospray emitter for analytes and an electrospray emitter for reagents, are positioned in a parallel fashion close to the sampling orifice of a triple quadrupole/linear ion trap tandem mass spectrometer (Sciex Q TRAP). The potentials applied to each sprayer are alternately pulsed so that ions of opposite polarity are generated separately in time. Ion/ion reactions take place after ions of each polarity are sequentially injected into a high-pressure linear ion trap, where axial trapping is effected by applying an auxiliary radio frequency voltage to the end lenses. The pulsed dual electrospray source allows optimization of each sprayer and can be readily coupled to any spray interface with no need for instrument modifications, provided the potentials required to transmit the ion polarity of interest can be alternated in synchrony with the emitter potentials. Ion/ion reaction examples such as charge reduction of multiply charged protein ions, charge inversion of peptides ions, and protein-protein complex formation are given to illustrate capabilities of the pulsed dual electrospray source in the study of gas-phase ion/ion chemistry. (J Am Soc Mass Spectrom 2005, 16, 1750-1756) @ 2005 American Society for Mass Spectrometry
\end{abstract}

$\mathrm{T}$ he advent of electrospray ionization (ESI) mass spectrometry has revolutionized the analysis of many biologically important macromolecules [1, 2]. Both the polarity and the magnitude of the charge associated with an ionized biomolecule play important roles in the use of tandem mass spectrometry for the identification and structural characterization of the biomolecule from which the ions were derived. Ion/ion reactions provide efficient means for manipulating the identities of gas-phase ions after their initial formation $[3,4]$, including altering ion polarity and absolute charge. Applications of charge manipulation via ion/ ion reactions include: mixture analysis [5-8], especially with the application of the "ion parking" technique for gas-phase concentration and charge state purification [9], the formation of ions that cannot be directly produced by ESI for subsequent tandem mass spectrometry studies [10], and the reduction of the product ion charge states to singly and doubly charged so as to simplify the interpretation of product ion spectra [1116]. Electron-transfer ion/ion reactions have recently been described as a means of inducing structurally informative dissociation, giving rise to cleavages analogous to those noted in electron capture dissociation [17-21]. In this regard, ion/ion reactions play a role as a probe of primary structure.

Electrodynamic ion traps, either a quadrupole ion

Published online September 22, 2005

Address reprint requests to Dr. S. A. McLuckey, Department of Chemistry, Purdue University, West Lafayette, IN 47907-2084, USA. E-mail: mcluckey@purdue.edu trap or a linear ion trap (LIT), are particularly useful reaction vessels for ion/ion reactions because of their ability to store oppositely-charged ions simultaneously [22] as well as their ion isolation and $\mathrm{MS}^{\mathrm{n}}$ functionalities [23]. Most ion/ion reaction studies involving multiply charged ions have been conducted with ion traps. In the vast majority of these studies, analyte and reagent ions have been generated by ion sources with distinct source/mass spectrometer interfaces, such as an ESI source for analyte ions and an atmospheric sampling glow discharge ionization (ASGDI) source for production of reagents for proton transfer [24] or electrontransfer [19] reactions, multiple ESI sources [25, 26] for complex formation [27-29] or charge increase [30, 31], and an ESI/chemical ionization (CI) combination for electron-transfer dissociation studies $[17,18]$. Since only one ion source interface is usually provided with a commercial instrument, implementing additional ionization interfaces has been required in the studies just mentioned. Recently, a single sonic spray source has been utilized for ion/ion reactions [32]. The advantage of using such an ion source is that instrument modification to add an additional ion source interface is obviated, because both polarities of ions can be produced simultaneously from a single sonic spray source. In that approach, analytes and reagents were mixed in one solution for the simultaneous formation of cationic and anionic reactants. Although no obvious signal suppression was found for certain pairs of reagents and analytes, the potential problem from matrix effects still exists for other mixtures, which may limit its use in the study of complex mixtures or samples with low abun- 
dance analytes. Furthermore, the ionization efficiency of sonic spray often does not compare favorably with other forms of spray ionization. Therefore, it is highly desirable to have a dual polarity ionization source that can be easily coupled to an instrument with minimal instrument modification and that allows for the independent optimization of ionization conditions for analyte and reagent species.

The use of two independent electrospray ionization sources, both of which were active at the same time, was first demonstrated by Smith et al. to generate the two polarities of ion beams [33, 34]. Ion/ion reactions took place at the base of a Y-tube outside a quadrupole mass spectrometer. This approach avoids extensive instrument modifications to accommodate an additional ion source interface. However, $\mathrm{MS}^{\mathrm{n}}$ experiments involving ion/ion reactions between stages of MS are not possible with this arrangement. Dual ESI sources or multiple sprayer systems have also been described and studied by different groups to improve electrospray sensitivity [35], to increase sample throughput [36], and to improve mass measurement accuracy in time-offlight (TOF), Fourier transform ion cyclotron resonance (FTICR), and sector mass spectrometry by providing a reference spray for internal mass calibration [37-39]. The dual or multiple sprayer systems usually operate in one of two ways. In one mode, all sprayers are active simultaneously and ions from all sprayers are sampled into the mass spectrometer. Under such a condition, depending upon the design, the simultaneous operation of two or more sprayers may lead to instabilities due to the interference of adjacent sprays [40], and the interaction of ions in the ESI interface can significantly reduce MS sampling efficiency [37, 41]. In the other typical operation mode, ions from distinct sprayers can be sampled sequentially by utilizing a rotatable plate [42] or rotor [43], which allow ions from a specific sprayer to enter the mass spectrometer sequentially. The mechanical switching may decrease the overall MS duty cycle and decrease sensitivity when compared to that of a single sprayer [44]. Other techniques have also been used for sequential sampling of ions, such as the application of an appropriate potential to an atmospheric pressure ion lens [36] and the alternate switching of voltages applied to the sprayers from a low potential to a high potential [40]. For all such applications, the high potentials applied to the sprayers have been of the same polarity.

This paper describes the application of a dual ESI source to the study of ion/ion reactions. In this work, a home-built dual electrospray source was positioned directly in front of a modified triple quadrupole/linear ion trap tandem mass spectrometer (i.e., a Sciex Q TRAP instrument) with axial trapping enabled by applying auxiliary radio frequency (RF) to the end lenses of one quadrupole array. Alternately pulsed high potentials were applied to the two spray emitters, which sequentially generated the oppositely charged ions for subsequent ion/ion reactions. Ion/ion reactions such as reducing multiply charged protein cations to singly charged ions, charge inversion of peptides, and proteinprotein complex formation are demonstrated with use of the pulsed dual electrospray source. The significance of the data lies in the convenience with which ion/ion reactions can now be implemented on an ion trap with minimal modification required. A single ion path can be used for both ion polarities and formation of the analyte ions and reagent ions can be optimized independently. This work emphasizes the combination of two independent sprayers. However, the overall approach can readily be extended to the use of more than two sprayers if a wider variety of reagent ions were desirable for some types of $\mathrm{MS}^{\mathrm{n}}$ experiments.

\section{Experimental}

\section{Materials}

Peptides and proteins were purchased from SigmaAldrich (St. Louis, MO) and used without further purification. Perfluoro-1-octanol (PFO) and dendrimers were obtained from Sigma-Aldrich (Milwaukee, WI). Solutions of peptides or proteins were dissolved to 5 $\mu \mathrm{M}$ in 50/50/1 (vol/vol/vol) methanol/water/acetic acid for positive ESI or in 50/50/2 (vol/vol/vol) methanol/water/ammonium hydroxide for negative ESI. PFO, with a final concentration of $100 \mu \mathrm{M}$, was subjected to negative ESI from $1 \%$ ammonium hydroxide methanol solution. A positive ESI spray solution of poly(propylenimine) [1,4-diaminobutane (DAB) core] dendrimers (generation 4 ) with a concentration of 0.5 $\mathrm{mg} / \mathrm{mL}$ was prepared in aqueous $5 \%$ acetic acid. A solution of carboxylate-terminated polyamidoamine (PAMAM) dendrimers (generation 1.5) was prepared in 50/50/2 (vol/vol/vol) methanol/water/ammonium hydroxide with a concentration of $1 \mathrm{mg} / \mathrm{mL}$ for negative electrospray.

\section{Apparatus Setup}

A home-built dual electrospray source was positioned directly in front of the nano-spray interface of a modified Sciex 2000 Q TRAP mass spectrometer (see below) as shown in Figure 1.

The two sprayers, typically a nano-electrospray emitter for analyte ion formation and an electrospray emitter for reagent ion formation, were clamped separately on a capillary holder. First, the position of the nanoelectrospray emitter was optimized with a distance 10-15 $\mathrm{mm}$ from the sampling orifice. The electrospray emitter was then placed in a parallel fashion, with its position (experimentally, 3-5 $\mathrm{mm}$ from the nano-electrospray emitter) manually adjusted to achieve the best sensitivity. The nano-electrospray solutions were loaded into nano-spray emitters with tip orifice diameter $30 \mu \mathrm{M}$, which were pulled from borosilicate capillaries (1.55 mm o.d., $0.86 \mathrm{~mm}$ i.d.) using a P-87 Flaming/Brown micropipette puller (Sutter Instruments, 


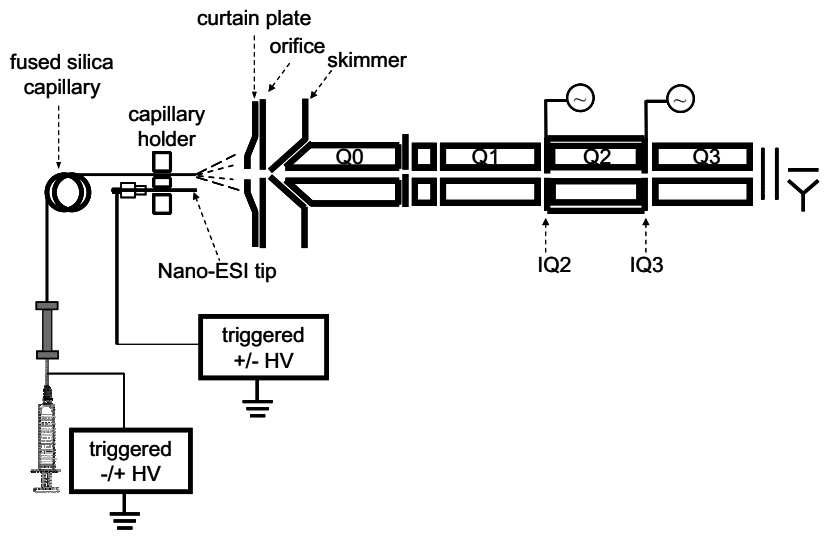

Figure 1. Diagram of the dual electrospray source couple with modified Q TRAP mass spectrometer.

Novato, CA). A stainless steel wire was inserted into the back of the capillary. The reagent solutions were introduced using a Harvard 11 syringe pump (Harvard Apparatus Inc., Boston, MA) at a flow rate of $1 \mu \mathrm{L} / \mathrm{min}$ into a fused silica capillary (100 $\mu \mathrm{m}$ i.d., $200 \mu \mathrm{m}$ o.d.). High voltages applied to each emitter were optimized separately and were independently generated by a high voltage power supply from the instrument and a fast high voltage pulser (GRX-1.5K-E, Directed Energy Inc., Fort Collins, CO). The two power supplies were triggered from the software, so that the high voltages were alternately switched on (off) in each scan. The switching intervals for the oppositely charged high voltage can be as short as $10 \mathrm{~ms}$; however, in the experiment it was set to $50 \mathrm{~ms}$ to allow injected ions to be collisionally cooled in the ion trap. The "off" voltages for each emitter were $0 \mathrm{~V}$ and the "on" voltages were between $\pm 1.2-2 \mathrm{kV}$ for the nano-electrospray emitter, and $\pm 3 \mathrm{kV}$ for electrospray. This pulsed source design enables stable spray from each emitter and allows ions of opposite charge to be generated and injected into the mass spectrometer separately.

All experiments were performed using a prototype version of a $Q$ TRAP mass spectrometer (Applied Biosystems/MDS SCIEX, Concord, Ontario, Canada) [45] that has been modified for ion/ion reactions [46]. (Note that the additional ion source adapted to the instrument, as described in [46] was not used in these studies.) All experiments were controlled by MS Expo 1.7.8 software provided by MDS SCIEX. The Q TRAP electronics were modified to allow superposition of an auxiliary RF signal to IQ2 and IQ3, which are the containment lenses of the Q2 quadrupole array. The auxiliary RF signal was optimized and set at $290 \mathrm{kHz}$, $100 \mathrm{~V}_{0-p}$ for each ion/ion reaction experiment.

For a typical ion/ion reaction experiment, high voltage on one emitter was pulsed on to generate one polarity of ions. Ions were isolated by Q1 in RF/DC mode and injected axially into the Q2 quadrupole array with nitrogen as buffer gas at a pressure of 6-8 mTorr. The ions were cooled in Q2 for $50 \mathrm{~ms}$, during which time the high voltage on this emitter was turned off. After this step, the oppositely charged high voltage on another emitter was triggered on, while the DC potentials applied to the ion path before Q2 were adjusted to allow ions of the other polarity to enter Q2 at relatively low kinetic energies (Q2 DC offset was about $5 \mathrm{~V}$ attractive to Q0 DC offset). During such a period, the DC potentials on IQ2 and IQ3, as well as on the Q2 rods themselves, were ramped to the same potential level while an auxiliary RF voltage was applied to IQ3. In the subsequent mutual ion storage step, the high potential on the second emitter was turned off, and the auxiliary RF signals were applied to both IQ2 and IQ3 to store ions axially. After a defined period of mutual storage, the unwanted polarity of ions was ejected from Q2 by applying attractive DC potentials to the Q2 containment lenses while the auxiliary RF signals applied to IQ2 and IQ3 were terminated. The product ions of interest were transferred from Q2 to Q3 and stored for $50 \mathrm{~ms}$ before they were subjected to mass selective axial ejection (MSAE) [47] using a supplementary RF signal at frequency $247 \mathrm{kHz}$, or at a frequency selected to give the desired mass range extension. The spectra shown here were typically the averages of 30-100 individual scans.

\section{Results and Discussion}

\section{Charge Reduction of Multiply Charged Positive Ions}

In many ion/ion reaction studies, charge reduction of multiply charged cations has been accomplished by reacting the cations with anions formed from glow discharge of perfluorocarbon compounds such as perfluoro-1,3-dimethylcyclohexane (PDCH) or perfluoro(methyldecalin) (PMD) [10, 48]. However, neither PDCH nor PMD are amenable to ionization via electrospray. Anions of perfluoro-1-octanol (PFO) formed from sonic spray ionization have recently been found to be efficient in reducing multiply charged protein/peptide ions with minimal tendency for adduct formation or for inducing fragmentation [32]. PFO was therefore employed in this study as a charge reducing reagent. In the dual electrospray source configuration, multiply charged protein/peptide cations were formed by positive nano-electrospray and anions derived from PFO were introduced by negative electrospray. As described in the experimental section, pulsed high voltages were applied to the two spray emitters such that ions of opposite polarity were generated separately in space and time. The advantage is that the interference from the adjacent sprays is greatly reduced since only one spray is on at a time. (No signal in either polarity was observed when both sprayers were on simultaneously.) The sequential nature of the dual sprayer also avoids the possibility for uncontrolled ion/ion reactions outside the mass spectrometer. Multiply charged cations of several different proteins and peptides were reacted with anions derived from PFO to test the performance 


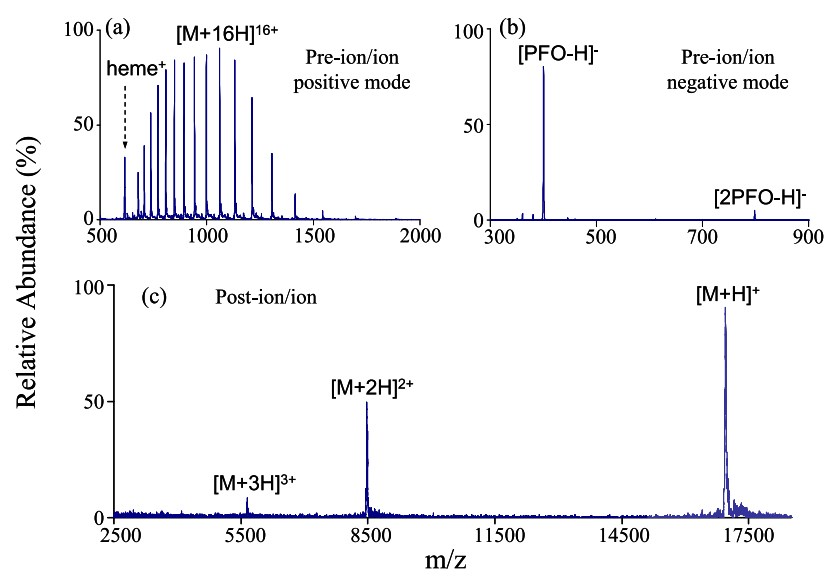

Figure 2. Mass spectra derived from the pulsed dual electrospray source: (a) positive nano-electrospray of $5 \mu \mathrm{M}$ horse heart myoglobin and (b) negative electrospray of $100 \mu \mathrm{M}$ PFO. (c) Post-ion/ion spectrum was acquired at $40 \mathrm{kHz}$ ejection frequency after $130 \mathrm{~ms}$ reaction of ions shown in (a) and (b).

of the pulsed dual electrospray source. Figure 2 gives an example of data relevant to the ion/ion reactions between cations formed from horse heart myoglobin and anions formed from PFO, which are both generated by the pulsed dual source.

The identities of the ions generated from each sprayer before ion/ion reaction are shown in Figure 2a and $\mathrm{b}$. The post-ion/ion mass spectrum (Figure 2c) was acquired at an axial ejection frequency of $40 \mathrm{kHz}$ after the entire myoglobin charge state distribution was subjected to ion/ion reaction with PFO anions ( $40 \mathrm{~ms}$ of injection) in the Q2 quadrupole array for $130 \mathrm{~ms}$. The $[\mathrm{M}+\mathrm{H}]^{+}$ions of myoglobin were most abundant in the product ion distribution, indicating ion/ion reaction rates very similar to those typically observed with the atmospheric sampling glow discharge ion source as an anion source. It is also noteworthy that the major anions formed in the negative electrospray of PFO are distinct from those formed via sonic spray ionization, where $[\mathrm{PFO}+\mathrm{Cl}]^{-}$anions are most abundant [32]. The latter ions can give rise to the formation of chloride transfer adducts to protein/peptide ions, which are, in general, undesirable because they can complicate the post-ion/ ion reaction spectra. Negative ion electrospray of PFO yields predominantly the deprotonated molecule and, to a lesser degree, the deprotonated dimer and therefore avoids the possibility for chloride adduct formation.

\section{Charge Inversion of Peptides}

When multiply charged reagent anions are employed, it is possible to invert the analyte ion charge. Charge inversion experiments in ion traps have been demonstrated to be useful in manipulating both ion charge and polarity in the gas-phase, and, when two sequential charge inversion reactions occur, can be used to increase the net charge of an ion in the gas-phase [30,31, 49]. So far, all such studies have been conducted with extensively modified instruments, in which two or three distinct electrospray interfaces were incorporated into the design $[25,26]$. Here, the pulsed dual electrospray source is demonstrated to implement charge inversion experiments using a single ion path and atmosphere/vacuum interface.

The data in Figure 3 demonstrate negative ion to positive ion charge inversion using the dual pulsed ESI arrangement for analyte and reagent ion formation. In this case, $[\mathrm{M}-\mathrm{H}]^{-}$ions of bradykinin were formed via nano-electrospray and transmitted through the Q1 quadrupole. Poly(propylenimine) 1,4-diaminobutane (DAB) dendrimer generation 4 cations were formed from positive electrospray with $[\mathrm{DAB}+7 \mathrm{H}]^{7+}$ ions being selected by Q1 for reaction. Figure $3 \mathrm{c}$ shows the ion/ion reaction products after mutual storage of the ions shown in Figure $3 \mathrm{a}$ and $\mathrm{b}$ for $200 \mathrm{~ms}$. The charge inversion products are obvious in Figure $3 \mathrm{c}$ with $[\mathrm{M}+$ $2 \mathrm{H}]^{2+}$ ions of bradykinin more abundant than the corresponding $[\mathrm{M}+\mathrm{H}]^{+}$ions. The relative abundances of $[\mathrm{M}+2 \mathrm{H}]^{2+}$ and $[\mathrm{M}+\mathrm{H}]^{+}$ions of bradykinin are dependent on several factors, including, in particular, the identity of the reagent ions (charge state, size, chemical functionalities). The use of the triple quadrupole platform is highly relevant in this regard because Q1 can be used to isolate independently the analyte and reagent ions. This is an advantage over in-trap ion isolation in that the ability to isolate ions of the second injected ion polarity is limited by the presence of the ions from the first injected ion polarity when ion trap selective ejection techniques are employed. Note that a small signal due to $[\mathrm{DAB}+6 \mathrm{H}]^{6+}$ is apparent in the pre-ion/ion spectrum, which arises due to ion/molecule proton transfer with background species within the Q2 LIT (nitrogen, 7 mTorr).

Figure 4 illustrates positive ion to negative ion charge inversion using the dual pulsed ESI arrange-

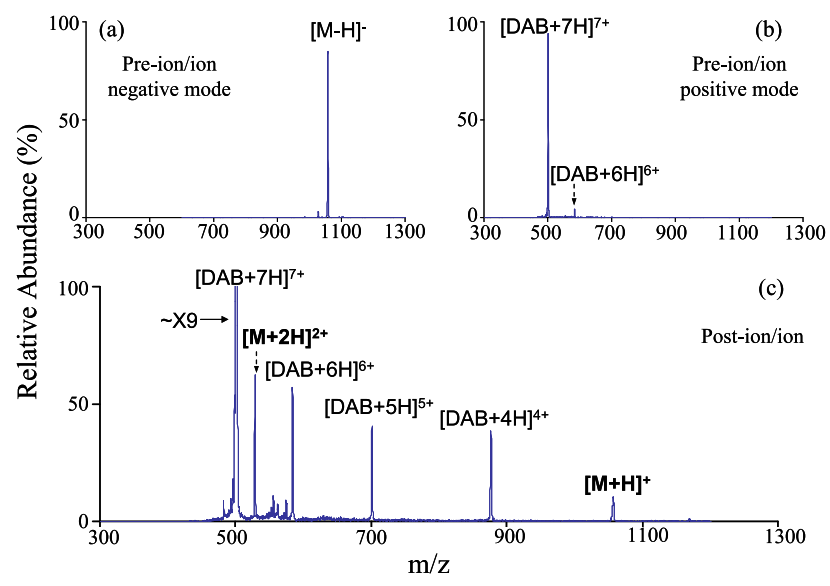

Figure 3. Mass spectra derived from the pulsed dual electrospray source: (a) isolated $[\mathrm{M}-\mathrm{H}]^{-}$ions of bradykinin from negative nano-electrospray and $(\mathbf{b})$ isolated $[\mathrm{DAB}+7 \mathrm{H}]^{7+}$ ions of DAB dendrimer generation 4 from positive electrospray. (c) Postion/ion spectrum was acquired after $200 \mathrm{~ms}$ reaction of ions shown in (a) and (b). 


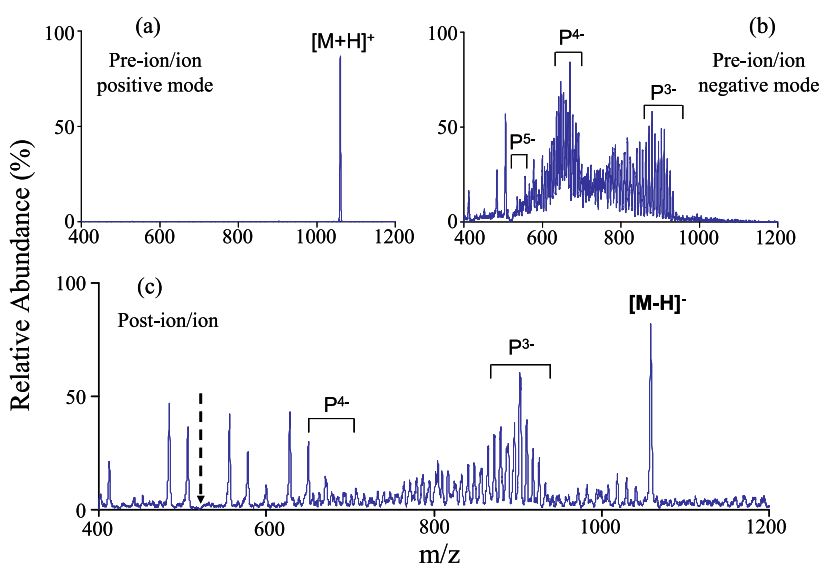

Figure 4. Mass spectra derived from the pulsed dual electrospray source: (a) isolated $[\mathrm{M}+\mathrm{H}]^{+}$bradykinin ions from positive nano-electrospray and $(\mathbf{b})$ negative electrospray of PAMAM dendrimer generation 1.5. (c) Post-ion/ion spectrum was acquired after $100 \mathrm{~ms}$ reaction of ions shown in (a) and (b). The arrow points where the $[\mathrm{M}-2 \mathrm{H}]^{2-}$ ions of bradykinin should be if they were present after ion/ion reactions.

ment for analyte and reagent ion formation. In this case, carboxylate-terminated polyamidoamine (PAMAM) dendrimer generation 1.5 was used to generate anions for charge inversion experiments. The PAMAM dendrimer 1.5 generation has an ethylene diamine core and an amidoamine repeating branching structure with carboxylate groups (sodium counter-ions) at the surface. In negative electrospray mode (Figure $4 \mathrm{~b}$ ), charge states of PAMAM dendrimers ranging from -3 to -5 were observed, denoted as $\mathrm{P}^{\mathrm{n}-}(n=3-5)$, as well as peaks arising from faulty synthesis products (not labeled in the spectrum). Within each charge state of PAMAM, mixtures of sodium ions and protons as counter-ions were present, which can be expressed as $[\mathrm{P}-(\mathrm{n}+\mathrm{m}) \mathrm{Na}+\mathrm{mH}]^{\mathrm{n}-}[\mathrm{m}=0-(16-\mathrm{n})]$. Since PAMAM dendrimer anion signal was spread over a wide variety of anionic species, no ion isolation via Q1 was employed so that the ion/ion reaction rate could be maximized. Therefore, $[\mathrm{M}+\mathrm{H}]^{+}$ions of bradykinin were subjected to reaction with the entire anion distribution derived from negative electrospray of PAMAM dendrimer generation 1.5. In the post-ion/ion spectrum of Figure $4 \mathrm{c},[\mathrm{M}-\mathrm{H}]^{-}$ions of bradykinin can be clearly identified, while the $[\mathrm{M}-2 \mathrm{H}]^{2-}$ ions of bradykinin (arrow pointed in Figure 4c) are not obvious. The formation of a highly abundant bradykinin dianion is not expected because bradykinin has only one strongly acidic site (viz., the C-terminus). When experiments shown in Figure 4 and Figure 3 are coupled by incorporating a third sprayer to the current dual sprayer system, charge increase experiments can readily be envisioned.

\section{Protein-Protein Complex Formation}

Ion/ion reactions have been shown to be capable of forming protein complexes in the gas-phase [27-29].
Comparing differences in dissociation behaviors between a complex formed in the gas-phase and nominally the same complex formed initially in solution, but then transferred to the gas-phase, can allow for inferences regarding the role of solvent, or lack thereof, in determining the gas-phase structure of the complex. A single sonic spray source has been used to form peptide-peptide complexes in earlier studies. The advantage to the sonic spray approach is that no modification was needed for the instrument to accommodate distinct ion source interfaces [32]. However, the ion/ion reaction complexes that can be generated via sonic spray are limited to the positive/negative ions which can be formed simultaneously from the same solution conditions. This limitation of using a single sonic spray source for complex formation can be successfully addressed with the use of the pulsed dual electrospray system. In the latter case, generation of a wider variety of positive and negative ions is possible for the reaction and, most importantly, each sprayer can be optimized independently. Data in Figure 5 demonstrate the capability of the pulsed dual electrospray source to form protein-protein complexes in the gas-phase. The $[\mathrm{M}+$ $8 \mathrm{H}]^{8+}$ ions of bovine ubiquitin, $\mathrm{U}^{8+}$, were isolated and reacted with the subsequently injected $[\mathrm{M}-4 \mathrm{H}]^{4-}$ anions of bovine insulin, denoted as $\mathrm{I}^{4-}$, in the Q2 LIT for $300 \mathrm{~ms}$. The predominant peak in the post-ion/ion spectrum is $(\mathrm{U}+\mathrm{I})^{4+}$, which is formed directly by the condensation of the two reactants. The $\mathrm{U}^{4+}, \mathrm{U}^{3+}$ and $\mathrm{I}^{+}$ products can arise from the breakup of the $(\mathrm{U}+\mathrm{I})^{4+}$ ion, while $\mathrm{U}^{7+}$ and $\mathrm{U}^{4+}$ ions can be formed from proton transfer reactions between $\mathrm{U}^{8+}$ and $\mathrm{I}^{4-}$ [28]. $\mathrm{U}^{7+}$ ions are present both in the pre-ion/ion spectrum (Figure 5a) and post-ion/ion spectrum (Figure $5 \mathrm{c}$ ). The formation of this ion is also attributed to ion/molecule proton transfer when ions are trapped in the high-pressure Q2 LIT. Due, at least in part, to the same reason, small signals corresponding to $(\mathrm{U}+\mathrm{I})^{3+}$ and $(\mathrm{U}+\mathrm{I})^{2+}$ are

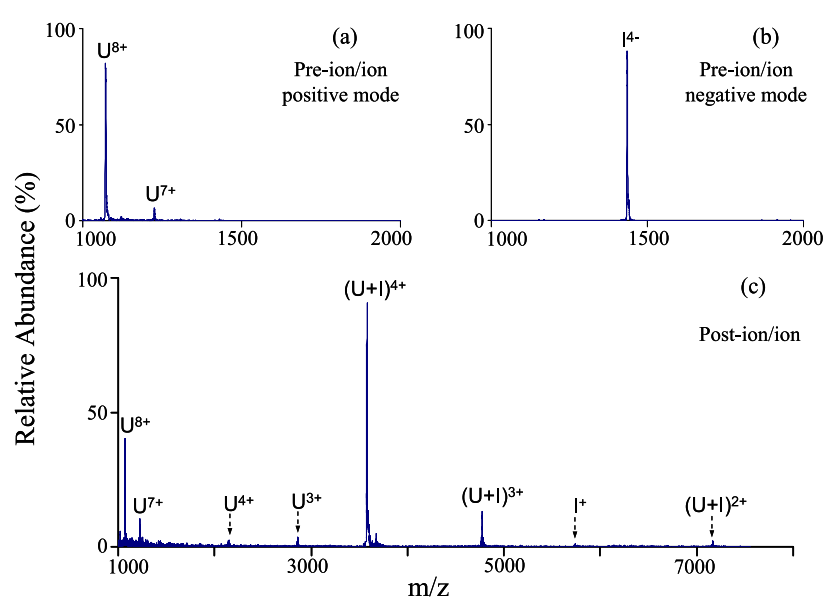

Figure 5. Mass spectra derived from the pulsed dual electrospray source: (a) isolated $[\mathrm{M}+8 \mathrm{H}]^{8+}$ ions of bovine ubiquitin from positive electrospray and (b) isolated $[\mathrm{M}-4 \mathrm{H}]^{4-}$ ions of bovine insulin from negative nano-electrospray. (c) Post-ion/ion spectrum was acquired after $300 \mathrm{~ms}$ reaction of ions shown in (a) and (b). 
also present in the post-ion/ion spectrum. In any case, this experiment demonstrates the formation of a protein/protein complex in the gas-phase from two species of protein ions which are formed sequentially by the dual electrospray source.

\section{Conclusions}

This paper provides proof of principle for the use of a pulsed dual electrospray ionization source to form reactants for subsequent ion/ion reactions. Compared to previously used methods for introduction of ionic reactants into electrodynamic ion traps, the advantages of the current source and analyzer design are severalfold: (1) the dual electrospray source can be directly coupled to any spray interface with minimum instrument hardware modifications required; (2) a high degree of control over the identities of the reactants, the reaction location, and reaction time are afforded by use of the quadrupole/LIT combination; (3) the dual electrospray source is much more versatile when compared to a single sonic spray ion source, since solution condition for each spray can be optimized independently; and (4) the dual electrospray source allows for the use of a nano-electrospray emitter, which is expected to be a significant advantage for analyte species present at relatively low levels. The efficacy of the dual electrospray ionization source was demonstrated in this paper with charge reduction, charge inversion, and complex formation ion/ion reactions. All experiments shown in this work were done in a linear ion trap which was modified to enable mutual trapping of oppositely charged ions in the axial direction. The same advantages are expected to accrue for coupling with threedimensional ion traps. A key requirement is that means are available to transmit ions of opposite polarity in synchrony with pulsing of the ion source. Given this key requirement, experiments need not be restricted to just two sprayers. For example, $\mathrm{MS}^{\mathrm{n}}$ experiments that require more than one type of reagent ion, such as the experiment to increase analyte ion charge via sequential charge inversion reactions, could conceivably be done with a three or more sprayer arrangement.

\section{Acknowledgments}

This work was sponsored by MDS SCIEX, an Industrial Associate of the Department of Chemistry. The authors acknowledge Dr. James W. Hager and Dr. Frank A. Londry of MDS SCIEX for helpful discussions and Adam Lau of MDS SCIEX for providing custom instrument control software. Technical support from Dr. Robert E. Santini of the Jonathan Amy Facility for Chemical Instrumentation is also appreciated.

\section{References}

1. Fenn, J. B.; Mann, M.; Meng, C. K.; Wong, S. F.; Whitehouse, C. M. Electrospray ionization for mass-spectrometry of large biomolecules. Science 1989, 246, 64-71.
2. Fenn, J. B.; Mann, M.; Meng, C. K.; Wong, S. F.; Whitehouse, C. M. Electrospray ionization principles and practice. Mass Spectrom. Rev. 1990, 9, 37-70.

3. McLuckey, S. A.; Stephenson, J. L., Jr. Ion/ion chemistry of high-mass multiply charged ions. Mass Spectrom. Rev. 1999, 18, 83-86.

4. Pitteri, S. J., McLuckey, S. A. Recent developments in the ion/ion chemistry of high-mass multiply charged ions. Mass Spectrom. Rev., in press.

5. Stephenson, J. L., Jr.; McLuckey, S. A. Ion/ion proton transfer reactions for protein mixture analysis. Anal. Chem. 1996, 68, 4026-4032.

6. Stephenson, J. L., Jr.; McLuckey, S. A. Charge manipulation for improved mass determination of high-mass species and mixture components by electrospray mass spectrometry. J. Mass Spectrom. 1998, 33, 664-672.

7. Pan, P.; McLuckey, S. A. Electrospray ionization of protein mixtures at low pH. Anal. Chem. 2003, 75, 1491-1499.

8. McLuckey, S. A.; Wu, J.; Bundy, J. L.; Stephenson, J. L., Jr.; Hurst, G. B. Oligonucleotide mixture analysis via electrospray and ion/ion reactions in a quadrupole ion trap. Anal. Chem. 2002, 74, 976-984.

9. McLuckey, S. A.; Reid, G. E.; Wells, J. M. Ion parking during ion/ion reactions in electrodynamic ion traps. Anal. Chem. 2002, 74, 336-346.

10. Stephenson, J. L., Jr.; McLuckey, S. A. Ion/ion reactions in the gas phase: Proton transfer reactions involving multiplycharged proteins. J. Am. Chem. Soc. 1996, 118, 7390-7397.

11. Wells, J. M.; Stephenson, J. L., Jr.; McLuckey, S. A. Charge dependence of protonated insulin decompositions. Int. J. Mass Spectrom. 2000, 203, A1-A9.

12. Reid, G. E.; Wu, J.; Chrisman, P. A.; Wells, J. M.; McLuckey, S. A. Charge state dependent sequence analysis of protonated ubiquitin ions via ion trap tandem mass spectrometry. Anal. Chem. 2001, 73, 3274-3281.

13. Newton, K. A.; Chrisman, P. A.; Reid, G. E.; Wells, J. M.; McLuckey, S. A. Gaseous apomyoglobin ion dissociation in a quadrupole ion trap: $[\mathrm{M}+2 \mathrm{H}]^{2+}-[\mathrm{M}+21 \mathrm{H}]^{21+}$. Int. J. Mass Spectrom. 2001, 212, 359-376.

14. Engel, B. J.; Pan, P.; Reid, G. E.; Wells, J. M.; McLuckey, S. A. Charge state dependent fragmentation of gaseous protein ions in a quadrupole ion trap: bovine ferri-, ferro-, and apocytochrome c. Int. J. Mass Spectrom. 2002, 219, 171-187.

15. Chrisman, P. A.; McLuckey, S. A. Dissociations of disulfidelinked gaseous polypeptide/protein anions: Ion chemistry with implications for protein identification and characterization. J. Proteome Res. 2002, 1, 549-557.

16. Hogan, J. M.; McLuckey, S. A. Charge state dependent collision-induced dissociation of native and reduced porcine elastase. J. Mass Spectrom. 2003, 38, 245-256.

17. Coon, J. J.; Syka, J. E. P.; Schwartz, J. C.; Shabanowitz, J.; Hunt, D. F. Anion dependence in the partitioning between proton and electron transfer in ion/ion reactions. Int. J. Mass Spectrom. 2004, 236, 33-42.

18. Syka, J. E. P.; Coon, J. J.; Schroeder, M. J.; Shabanowitz, J.; Hunt, D. F. Peptide and protein sequence analysis by electron transfer dissociation mass spectrometry. Proc. Natl. Acad. Sci. U.S.A. 2004, 101, 9528-9533.

19. Pitteri, S. J.; Chrisman, P. A.; Hogan, J. M.; McLuckey, S. A. Electron transfer ion/ion reactions in a three-dimensional quadrupole ion trap: Reactions of doubly and triply protonated peptides with $\mathrm{SO}_{2}^{--}$. Anal. Chem. 2005, 77, 1831-1839.

20. Chrisman, P. A.; Pitteri, S. J.; Hogan, J. M.; McLuckey, S. A. $\mathrm{SO}_{2}^{-}$electron transfer ion/ion reactions with disulfide linked polypeptide ions. J. Am. Soc. Mass Spectrom. 2005, 16, 1020-1030. 
21. Hogan, J. M.; Pitteri, S. J.; Chrisman, P. A.; McLuckey, S. A. Complementary structural information from a tryptic $\mathrm{N}$ linked glycopeptide via electron transfer ion/ion reactions and collision-induced dissociation. J. Proteome Res. 2005, 4, 628-632.

22. Mather, R. E.; Todd, J. F. J. The quadrupole ion store (Quistor). 7. Simultaneous positive-negative ion mass-spectrometry. Int. J. Mass Spectrom. Ion Phys. 1980, 33, 159-165.

23. McLuckey, S. A.; Glish, G. L.; Van Berkel, G. J. Multiple stages of mass-spectrometry in a quadrupole ion trap mass-spectrometer-prerequisites. Int. J. Mass Spectrom. Ion Processes 1991, 106, 213-235.

24. Stephenson, J. L., Jr.; McLuckey, S. A. Adaptation of the Paul trap for study of the reaction of multiply charged cations with singly charged anions. Int. J. Mass Spectrom. Ion Processes 1997, 162, 89-106.

25. Wells, J. M.; Chrisman, P. A.; McLuckey, S. A. “Dueling” ESI: Instrumentation to study ion/ion reactions of electrospraygenerated cations and anions. J. Am. Soc. Mass Spectrom. 2002, $13,614-622$.

26. Badman, E. R.; Chrisman, P. A.; McLuckey, S. A. A quadrupole ion trap mass spectrometer with three independent ion sources for the study of gas-phase ion/ion reactions. Anal. Chem. 2002, 74, 6237-6243.

27. Wells, J. M.; Chrisman, P. A.; McLuckey, S. A. Formation of protein-protein complexes in vacuo. J. Am. Chem. Soc. 2001, 123, 12428-12429.

28. Wells, J. M.; Chrisman, P. A.; McLuckey, S. A. Formation and characterization of protein-protein complexes in vacuo. J. Am. Chem. Soc. 2003, 125, 7238-7249.

29. Gunawardena, H. P.; McLuckey, S. A. Synthesis of multi-unit protein hetero-complexes in the gas phase via ion-ion chemistry. J. Mass Spectrom. 2004, 39, 630-638.

30. He, M.; McLuckey, S. A. Increasing the negative charge of a macroanion in the gas phase via sequential charge inversion reactions. Anal. Chem. 2004, 76, 4189-4192.

31. He, M.; McLuckey, S. A. Two ion/ion charge inversion steps to form a doubly protonated peptide from a singly protonated peptide in the gas phase. J. Am. Chem. Soc. 2003, 125, 77567757.

32. Xia, Y.; Liang, X.; McLuckey, S. A. Sonic spray as a dual polarity ion source for ion/ion reactions. Anal. Chem. 2005, 77, 3683-3689.

33. Ogorzalek-Loo, R. R.; Udseth, H. R.; Smith, R. D. Evidence of charge inversion in the reaction of singly charged anions with multiply charged macroions. J. Phys. Chem. 1991, 95, 64126415.

34. Ogorzalek-Loo, R. R.; Udseth, H. R.; Smith, R. D. A new approach for the study of gas-phase ion-ion reactions using electrospray ionization. J. Am. Soc. Mass Spectrom. 1992, 3, 695-705.

35. Tang, K.; Lin, Y.; Matson, D. W.; Kim, T.; Smith, R. D. Generation of multiple electrosprays using microfabricated emitter arrays for improved mass spectrometric sensitivity. Anal. Chem. 2001, 73, 1568-1663.
36. Shneider, B. B.; Douglas, D. J.; Chen, D. D. Y. Multiple sprayer system for high-throughput electrospray ionization mass spectrometry. Rapid Commun. Mass Spectrom. 2002, 16, 1982-1990.

37. Jiang, L.; Moini, M. Development of multi-ESI-sprayer, multiatmospheric-pressure-inlet mass spectrometry and its application to accurate mass measurement using time-of-flight mass spectrometry. Anal. Chem. 2000, 72, 20-24.

38. Hannis, J. C.; Muddiman, D. C. A dual electrospray ionization source combined with hexapole accumulation to achieve high mass accuracy of biopolymers in Fourier transform ion cyclotron resonance mass spectrometry. J. Am. Soc. Mass Spectrom. 2000, 11, 876-883.

39. Takahashi, Y.; Fujimaki, S.; Kobayashi, T.; Morita, T.; Higuchi, $\mathrm{T}$. Accurate mass determination by multiple sprayers nanoelectrospray mass spectrometry on a magnetic sector instrument. Rapid Commun. Mass Spectrom. 2000, 14, 947-949.

40. Satomi, Y.; Kudo, Y.; Sasaki, K.; Hase, T.; Takao, T. Accurate mass measurement in nano-electrospray ionization mass spectrometry by alternate switching of high voltage between sample and reference sprayers. Rapid Commun. Mass Spectrom. 2005, 19, 540-546.

41. Zhou, F.; Shui, W.; Lu, Y.; Yang, P.; Guo, Y. High accuracy mass measurement of peptides with internal calibration using a dual electrospray ionization sprayer system for protein identification. Rapid Commun. Mass Spectrom. 2002, 16, 505511.

42. Wolff, J.; Eckers, C.; Sage, A. B.; Giles, K.; Bateman, R. Accurate mass liquid chromatography/mss spectrometry on quadrupole orthogonal acceleration time-of-flight mass analyzers using switching between separate sample and reference sprays. 2. Applications using the dual-electrospray ion source. Anal. Chem. 2001, 73, 2605-2612.

43. Nepomuceno, A. I.; Muddiman, D. C.; Bergen, H. R., III; Craighead, J. R.; Burke, M. J.; Caskey, P. E.; Allan, J. A. Dual electrospray ionization source for confident generation of accurate mass tags using liquid chromatography Fourier transform ion cyclotron resonance mass spectrometry. Anal. Chem. 2003, 75, 3411-3418.

44. Yang, L.; Mann, T. D.; Little, D.; Wu, N.; P., C. R.; Rudewicz, P. J. Evaluation of a four-channel multiplexed electrospray triple quadrupole mass spectrometer for the simultaneous validation of LC/MS/MS methods in four different preclinical matrixes. Anal. Chem. 2001, 73, 1740-1747.

45. Hager, J. W. A new linear ion trap mass spectrometer. Rapid Commun. Mass Spectrom. 2002, 16, 512-526.

46. Wu, J.; Hager, J. W.; Xia, Y.; Londry, F. A.; McLuckey, S. A. Positive ion transmission mode ion/ion reactions in a hybrid linear ion trap. Anal. Chem. 2004, 76, 5006-5015.

47. Londry, F. A.; Hager, J. W. Mass selective axial ion ejection from a linear quadrupole ion trap. J. Am. Soc. Mass Spectrom. 2003, 14, 1130-1147.

48. Xia, Y.; Wu, J.; McLuckey, S. A.; Londry, F. A.; Hager, J. W. Mutual storage mode ion/ion reactions in a hybrid linear ion trap. J. Am. Soc. Mass Spectrom. 2005, 16, 71-81.

49. He, M.; Emory, J. F.; McLuckey, S. A. Reagent anions for charge inversion of polypeptide/protein cations in the gas phase. Anal. Chem. 2005, 77, 3173-3182. 